\title{
Notas acerca de los estudios judaicos en chile
}

Notes about jewish studies in Chile

Ana María Tapia Adler

amtuch@u.uchile.cl

Universidad de Chile

\section{Resumen}

El presente artículo pretende entregar una visión panorámica de cómo se presenta el estado de los estudios judaicos en Chile, tanto en el ámbito académico como fuera de él y las áreas en las que se ha trabajado tanto en investigación como en Seminarios de Tesis en universidades chilenas.

Palabras claves: judíos, inmigrantes en judíos, judaísmo, judíos en Chile, memoria judía, estudios judaicos, judaísmo latinoamericano.

\begin{abstract}
This article aims to provide a panoramic view of how the state of Jewish studies in Chile is presented, both in the academic field and outside of it, and the areas in which research and seminary work have been carried out in Chilean universities.
\end{abstract}

Keywords: Jews, Judaism, Jews in Chile, Latinamerican Judaism. 
Los estudios de judaísmo latinoamericano a nivel universitario en Chile son de antigua data. Principalmente desarrollados por académicos pertenecientes al Centro de Estudios Judaicos (CEJ) de la Universidad de Chile, entidad fundada en el año 1968.

Hubo dos grandes investigadores acerca de esta temática, Günter Böhm (zl) (Chile), dedicado casi exclusivamente a los siglos XIX y anteriores y que fuera Director del CEJ, y Moshé Nes $\mathrm{El}^{1}$ (zl), chileno-israelí, que abarcó temáticas del siglo XX. El trabajo de estos dos personajes dio origen a una vasta bibliografía y presentaciones en diversos congresos. AMILAT, la Asociación Israelí para la investigación del Judaísmo Latinoamericano, dedicó a Moshé Nes-El su última publicación de Judaica Latinoamericana, recientemente presentada en el XVII Congreso Mundial de Estudios Judaicos llevado a cabo en la Universidad Hebrea de Jerusalén entre el 6 y el 10 de agosto del presente año (2017). Nos permitimos no referirnos a la vasta producción de ambos investigadores, a fin de no alargar este escrito y por considerar que son ya conocidos por el amplio público.

El presente escrito se propone presentar el estado de los Estudios judaicos en Chile, lo que haremos en base a las preguntas orientadoras sobre las cuales se trabajó en la mesa "Los estudios judíos desde América Latina", en el XVII Congreso Internacional de LAJSA, realizado en Miami en mayo de 2015, coordinada por Emmanuel Kahn. Dicha instancia permitió a quienes participaron, cuestionarse acerca de las siguientes interrogantes orientadoras:

1) ¿Cuáles son las diversas líneas de trabajo e investigación acerca de los judíos que se realizan en los países de los expositores?

2) ¿En qué medida esos temas dialogan, se inscriben y/o son subsidiarios de la agenda de debates académica local?

3) ¿Qué rasgos permiten observar el modo en que lo "judío" dialoga con otros colectivos sociales y nacionales?

\footnotetext{
${ }^{1}$ En http://moshe.nes-el.com/spanish.php el lector interesado podrá encontrar artículos, cuentos y poemas, libros, revistas y periódicos de este notable historiador y escritor.
} 
4) ¿Cuáles son las vías de “institucionalización” que atraviesan los estudios judíos en cada uno de los países de los participantes del panel?

En base a las preguntas anteriormente planteadas, que intentaran ser respondidas, no necesariamente en el mismo orden, y para los efectos de poder entregar información acerca de lo que ocurre en el área que nos ocupa, se realizó en ese año una pesquisa, consultando a Directores y Decanos de distintas universidades acerca de la existencia de académicos que se dedicasen a esta temática, en cualquiera de las disciplinas que se desarrollan en sus universidades y que tocaran el tema en cuestión.

De las respuestas recibidas se pudo colegir que, salvo en casos especiales en que se tocan temas de migración, la temática de judaísmo latinoamericano no ha sido un tema preferente en las distintas universidades. Al menos no en el ámbito de las ciencias sociales, con excepción, principal, aunque no exclusivamente, de trabajos eventuales de tesis de alumnos especialmente de pregrado.

Establecido lo anterior, empezaremos, a continuación a señalar lo que pudimos encontrar gracias a la pesquisa realizada.

En el ámbito de la literatura, es posible encontrar algunas publicaciones, entre las que pueden citarse, por ejemplo, la obra Voces judías de la literatura chilena de Rodrigo Cánovas y Jorge Scherman y, sin publicar aún, se encuentra la tesis doctoral de Juana Campos sobre La errancia judía en la novela contemporánea hispanoamericana.

En relación con la primera de las obras mencionadas, puede señalarse que abarca a diecisiete autores cuyas temáticas se centran en la identidad judía, abordadas desde diferentes géneros literarios, en los que se encuentra la imagen del inmigrante llegado a Chile, país al que hicieron su hogar y encontraron la paz que buscaban arrancando de las difíciles condiciones de vida en sus lugares de origen. 
La segunda investigación, que corresponde a la tesis doctoral de la Prof. Campos, se centra en el tema de la errancia, una constante en la historia judía que ha sido tratada en distintas novelas hispanoamericanas, de entre las cuales la autora ha escogido cuatro obras, a saber, La vida a plazos, de don Jacobo Lerner de Isaac Goldemberg, Morirás lejos de José Emilio Pacheco, Las genealogías de Margo Glantz y La gesta del marrano de Marcos Aguinis. El objetivo de esta tesis doctoral era realizar una sistematización de las referencias que se hacen del judaísmo en orden a describir los modos en que se diseña la figura de la errancia, que es la columna vertebral de su investigación. La Doctora Campos se desempeñó durante varios años como académica del Centro de Estudios Judaicos de la Universidad de Chile, donde se dedicó principal, aunque no exclusivamente, a la temática de la literatura y multiculturalidad judía.

Marjorie Agosin, activista de los DD.HH. y académica del Wellesley College es otra de las escritoras que ha tocado el tema, aunque más bien tangencialmente, en su libro Una cruz y una estrella: las memorias de una muchacha judía en Chile. La familia de Marjorie emigró a Estados Unidos en 1968, cuando ella contaba 13 años.

Existen obras de escritores de origen judío que no necesariamente tocan el tema del judaísmo latinoamericano como tal, pero que en alguna medida la figura del inmigrante llegado a estos parajes está presente: Es el caso de Sonia Guralnik, quien llegó a Chile junto a su familia, proveniente de Kiev, y recrea, a partir de su propia experiencia, la vida de los judíos rusos llegados a nuestro país en los años 30. De ella se conocen cuentos que grafican en forma vívida recuerdos de costumbres y tradiciones que se mantuvieron en su familia y que llenan de nostalgia sus escritos. Entre sus publicaciones destacan El Samovar, Para siempre en mi memoria, Sonata de carne y hueso. En una entrevista concedida al Diario Las Últimas Noticias (sábado 26 de mayo de 2001) la autora señaló: "Soy muy modesta con lo que escribo. Siento que tengo aún mucho por aprender. Y, sobre todo, me gusta que sea una literatura positiva”.

Presentes también en la escena literaria actual se encuentran los escritores Eliah Germani, Carla Guelfenbeim, Volodia Teitelboim, Cynthia Rimsky, Andrea Jeftanovich, y, mucho 
más recientemente, Hernán Rodriguez Fisse, hijo de inmigrantes judíos provenientes de Turquía, ganador del Premio Revista de Libros 2016, del Diario El Mercurio, con su libro Prefiero Chile.

Desde la perspectiva de la ciencia política y la sociología destaca el trabajo serio y constante de Isaac Caro, Doctor en Estudios Americanos con mención en Relaciones Internacionales, y académico de la Universidad Alberto Hurtado cuya temática principal está centrada sobre Fundamentalismos en América Latina, principalmente sobre fundamentalismos islámico y judío. También incursiona con éxito en el tema del dialogo de civilizaciones.

Entre las publicaciones de este investigador destacan Islam y Judaísmo contemporáneos en América Latina, trabajo que abarca tres países del Cono Sur: Argentina, Brasil y Chile, Fundamentalismos islámicos. Guerra contra Occidente y América Latina; Extremismos de derecha y movimientos neonazis. Islam y judaísmo contemporáneo en América Latina. Los casos de Argentina, Brasil y Chile. Todos ellos producto de sendas investigaciones financiadas por CONICYT. Caro, además, cuenta con una serie de capítulos de libros y artículos sobre la temática. Entre ellos, Miradas y mensajes desde la globalización: el caso del antisemitismo y la xenofobia, Identidades judías y sionistas en conflicto. Diásporas judías en el Cono Sur americano ${ }^{2}$, Conflictos de identidades y política internacional, Conflicto y pacificación en las relaciones entre las comunidades judías, árabes y musulmanas en Chile y Argentina ${ }^{3}$. Ciertamente el de Isaac Caro es un trabajo contundente, serio y constante en el tiempo.

Explorando en el mundo de las tesis se abre ante el espectador un amplio espectro. En la temática de las relaciones entre los judíos y el conflicto en el Medio Oriente se encuentra la tesis de licenciatura para optar al título de antropóloga social de Maia Guiskin, Ser Judío en

${ }^{2}$ Identidades judías y sionistas en conflicto. Diásporas judías en el Cono Sur americano, en Gilberto Aranda y Sergio Salinas (editores).

${ }^{3}$ En Lorenzo Agar y Abraham Magendzo: Diálogo intercultural. Caso Comunidad Judía y árabe en Chile. Santiago: Fundación Ideas, Proyecto DIJO, pp. 185-212. ISBN: 978- 956-7597-06-2. Proyecto FONDECYT No 1050053. 
Chile, Identidad y conflicto palestino-israelí (2013) ${ }^{4}$. Como muy bien explica la autora su investigación es una:

Reflexión en torno a la identidad judía en Chile a partir de las tensiones que genera el conflicto palestino-israelí para los judíos chilenos. La realización de su trabajo a través de técnicas conversacionales aplicadas en distintos sectores de la comunidad judía chilena, se distinguieron las opiniones y posicionamientos que se generan en torno al conflicto palestino-israelí, identificando los elementos que definen y tensionan la identidad judía en Chile (p.6).

Desde la perspectiva del antisemitismo encontramos una amplia bibliografía, principalmente artículos y un par de tesis de grado como la de Gustavo Guzmán Castro que obtuvo su grado de Licenciado en Historia con el tema sobre El Nazismo, religión de violencia, a partir de la cual se interesó vivamente en la temática del antisemitismo en Chile, tanto es así que su tesis de magistratura versó sobre La patria sinjudios: antisemitismo nacionalista en Chile, 1932-1940: los casos del Movimiento Nacional Socialista y del Partido Nacional Fascista (2012) ${ }^{5}$. Del mismo modo, Guzmán ha publicado artículos en la revista Cuadernos Judaicos que dan razón, principalmente, de la temática relacionada con el autor antisemita más famoso de Chile, Miguel Serrano y el movimiento nacionalsocialista ${ }^{6}$ en nuestro país. Judeofobia y mito conspirativo en la obra de Miguel Serrano ${ }^{7}$, Respuestas de la prensa antifascista al antisemitismo del Partido Nacional Fascista de Chile (1938-1940) ${ }^{8}$, Chile para todas las razas, Al margen del antisemitismo: Respuestas del semanario Mundo Judio al Movimiento Nacional Socialista

\footnotetext{
${ }^{4}$ Facultad de Ciencias Sociales, Universidad de Chile, 2013.

${ }^{5}$ Tesis para optar al grado de Magister en Historia, Depto. de Historia. Facultad de Filosofía y Humanidades, Universidad de Chile, 2012, Universidad de Chile.

${ }^{6}$ Partido Naci, con "c", cosa que se recalca para señalar que no es lo mismo que nazi.

${ }^{7}$ Cuadernos Judaicos Núm. 26 (2009): Diciembre

${ }^{8}$ Cuadernos Judaicos Núm. 32 (2015): Diciembre
} 
de Chile (1935-1938) ${ }^{9}$, Judeofobia en Chile durante la década del ochenta: la obra de Miguel Serrano ${ }^{10}$, Negación del Holocausto en Chile: Miguel Serrano en los años ochenta $^{11}$, Ironizando con el antisemitismo: respuestas del semanario Mundo Judio al Partido Nacional Fascista de Chile $(1938-1940)^{12}$, Judeofobia y mito conspirativo en la obra de Miguel Serrano ${ }^{13}$. Guzmán Castro se encuentra en la actualidad cursando sus estudios de doctorado en la Universidad de Tel Aviv, bajo la tutoría de Raanan Rein.

En la misma línea se encuentran artículos del académico chileno-israelí, de la Universidad Hebrea de Jerusalem, Mario Sznajder, algunos de ellos son: El nacional socialismo chileno de los años treinta ${ }^{14}$, El Movimiento Nacional Socialista: nacismo a la chilena ${ }^{15}$, The Antisemitic Myth and National Socialism in Chile, ${ }^{16}$, O Anti-semitismo no Chile ${ }^{17}$. En el espectro político partidario destacan sus artículos Una comunidad en crisis: el judaísmo chileno y el gobierno de la Unidad Popular (1993), referido a la época en la que "se produjo un cierto recrudecimiento del antisemitismo, especialmente por parte de la extrema derecha política chilena y en mucho menor grado por parte de sectores de izquierda" ${ }^{\text {18 }}$. La bibliografía de Sznajder es mucho más amplia, pero acá mencionamos solo lo relacionado con Chile, aun cuando estos títulos pudieran no ser los únicos producidos por este investigador sobre la materia pero sí los que se encuentran a nuestro alcance.

De Valeria Navarro-Rosenblatt destacan La dirigencia judía en el periodo de la dictadura, Discriminación y reconciliación comunidad Judeo-Argentina y su relación con el Régimen Militar Argentino (1976-1989) ${ }^{19}$, Ampliando los límites de la integración social: género e

\footnotetext{
${ }^{9}$ Cuadernos Judaicos Núm. 30 (2013): Diciembre

${ }^{10}$ Cuadernos Judaicos Núm. 25 (2008): Diciembre

${ }^{11}$ Cuadernos Judaicos Núm. 27 (2010): Diciembre

${ }^{12}$ Cuadernos Judaicos Núm. 31 (2014): Diciembre

${ }^{13}$ Cuadernos Judaicos Núm. 26 (2009): Diciembre

${ }^{14}$ M. Sznajder - Mapocho, 1992.

${ }^{15}$ Estudios Interdisciplinarios de América Latina y el Caribe $(1,1,1990)$ pp. 41-57.

${ }^{16}$ Yahadut Zmaneinu $(7,1992)$ pp. 311-327. (Hebrew).

${ }^{17}$ En M. L. Tucci Carneiro (ed.) O Anti-semitismo nas Américas (Sao Paulo: Editora EDUSP, 2008) pp. $437-457$

${ }_{18}$ Judaica Latinoamericana $(2,1993)$ pp. 137-148.

${ }^{19}$ Cuadernos Judaicos Núm.26 (2009): Diciembre
} 
historia judía contemporánea $a^{20}$, además de su tesis de Magistratura sobre Comunidad judía en Chile y Argentina durante los regímenes militares 1973-1990/1976-1983: dirigencia y DD:HH, The Untold History: Voices of Non-affiliated Jews in Chile, 1940$1990^{21}$, y su posterior tesis de doctorado en la Universidad de Wisconsin-Madison (USA), Comunistas judíos en Chile: Experiencia y memoria en las historias de vida de Dora Guralnik y Carlos Berger (1930-1990).

Cuadernos Judaicos, revista electrónica del Centro de Estudios Judaicos, es un repositorio de trabajos que abordan el judaísmo y temáticas anexas, podemos encontrar dos artículos de Mario Matus González, ex profesor el CEJ y actual Director del Departamento de Historia de nuestra Facultad: Verdad histórica y perdón: el caso alemán, 1945-2009 22 en este escrito el autor sitúa el tema del perdón en Alemania desde el periodo de la postguerra hasta la actualidad. En él analiza las consecuencias de la relación entre culpa y justicia y la opción de borrar la memoria colectiva durante un período prolongado y extrae algunos aprendizajes que podrían reportar cierto interés para el proceso chileno de recuperación de la justicia y la memoria histórica y Trayectoria de la usura y el lucro en el judaísmo ${ }^{23}$ que si bien no está referido directamente a judaísmo latinoamericano, el tema está presente en el imaginario colectivo que se tiene en Chile y el prejuicio sobre los judíos y el dinero. El artículo en cuestión "sigue la evolución de la actitud hacia el préstamo de dinero con interés dentro del mundo judío, inserta dentro de una sucesión de pronunciamientos de muchas civilizaciones del mundo antiguo y antes de que el Cristianismo medieval irrumpiera con una posición condenatoria extrema" (Matus, 2013)

Matus junto con Tapia-Adler, dirigieron el Seminario de Tesis titulado Reglas, capacidades y redes en el desempeño económico de familias judías en Chile: 1930-2009, producto del

\footnotetext{
${ }^{20}$ Valeria Navarro-Rosenblatt, Ampliando los límites de la integración social: género e historia judía contemporánea, Cuadernos Judaicos, Centro de Estudios Judaicos, Universidad de Chile, 30 (2013).

${ }^{21}$ The Untold History: Voices of Non-affiliated Jews in Chile, 1940-1990 (2017) ${ }^{21}$, in Raanan Rein ed. New Ethnic Studies, Brill editorial.

${ }^{22}$ Cuadernos Judaicos Núm. 28 (2011): Diciembre

${ }^{23}$ Cuadernos Judaicos Núm. 30 (2013): Diciembre
} 
cual destacan las tesis de grado de Jorge Vicencio Meza, sobre Capital Institucional y Desarrollo Económico: Una aproximación a la relación normativa-conductual en comunidades extranjeras. Empresarios Judíos en Chile. 1930-1970; de Tania López Sepúlveda, Influencias familiares en la formación del empresariado judío: Chile, 19302009 y Paulina Silva Peñaloza, Capital Social en Familias Judías, Chile 1930-2009.

Otro tema presente en trabajos de investigación que han dado origen a libros y artículos tiene que ver con Migración. Así es como destacan la Tesis para optar al título de Licenciado en Historia con Mención en Estudios Culturales de Vicente Hernández Ferrada, egresado de la Universidad Academia de Humanismo Cristiano, cuyo título es Refugiados indeseables en Chile antes y durante la Segunda Guerra Mundial. El carácter antisemita de los nazis y nacis chilenos y sus aliados en la derecha liberal y conservadora, (2015). Este trabajo está dedicado a "aquellos personajes anónimos, que supieron oponerse a los apologistas del odio totalitario y sus amenazas de retrotraer la historia a tiempos pretéritos, de odios y persecuciones raciales" (p. 5). Su trabajo abarca una época en la que el ambiente político del país se encontraba sumamente convulsionado y en el que surgieron los primeros movimientos y partidos de corte fascista y nazista. En relación con el convulsionado ambiente político y social de aquel periodo señala que "surgieron los primeros movimientos y partidos que pretendieron emular a los fascistas y nazistas europeos, el primero de ellos fue la Legión Social Nacionalista del ex Inspector del Ejército y general retirado Francisco Díaz Valderrama en junio de 1932 que predicaba un antisemitismo radical..." (p. 9).

El académico de la Universidad de Los Andes, Enrique Brahm García, ha trabajado sobre el tema y encontramos dos muy interesantes artículos relacionados con migración. Ellos son: El Frente Popular y la inmigración judía a Chile: de la apertura al cierre total ${ }^{24}$, y

\footnotetext{
${ }^{24}$ Brahm, G., Enrique y Montes Arraztoa, Jorge. El frente popular y la inmigración judía a chile: de la apertura al cierre total. Rev. chil. derecho [online]. 2012, vol.39, n.3 [citado 2017-12-14], pp.891-907. Disponible en:
} 
Obstáculos jurídicos y consulares a la inmigración judía durante el gobierno de Arturo Alessandri 1932-1938 ${ }^{25}$. El primero de estos artículos está basado en circulares del Ministerio de Relaciones Exteriores analiza la normativa del estatuto "por el cual se sometió a reglas especiales, que no eran válidas para el común de los inmigrantes, a los judios que quisieran trasladarse a Chile ${ }^{, 26}$, el Frente Popular, en cambio, no estaba de acuerdo dichas normas restrictivas por lo que buscaba una mayor flexibilización de la normativa. En el segundo artículo, el autor en el abstract, señala:

Cuando en la Alemania nazi se inició un proceso de discriminación y de persecución contra la población judía, que provocó un masivo proceso migratorio, las puertas de la mayoría de los países de Occidente no se abrieron con generosidad para acoger a estos perseguidos. Chile no fue una excepción. Desde muy temprano el gobierno de Arturo Alessandri puso trabas a la inmigración judía, merced a un estatuto jurídico especial, discriminatorio, que se hizo más duro en los años finales de su período presidencial. La revisión de la correspondencia consular en el Archivo del Ministerio de Relaciones Exteriores, demuestra que también en Chile había quienes sostenían posiciones antisemitas más radicales, a tono con la época ${ }^{27}$.

Están también los artículos El viaje inmigratorio de familias judías hacia Chile en el siglo $X X^{28}$ de Matus donde describe los principales aspectos del viaje inmigratorio de familias judías a Chile e Inmigración judía a Chile desde la Alemania Nazi de la escritora Eva Goldschmidt en donde narra las dificultades que enfrentaron para la http://www.scielo.cl/scielo.php?script=sci_arttext\&pid=S0718372012000300020\&lng=es\&nrm=iso>. ISSN 0718-3437. http://dx.doi.org/10.4067/S071834372012000300020.

${ }^{25}$ Brahm, G., Enrique y Montes Arraztoa, Jorge. (2013). Obstáculos jurídicos y consulares a la inmigración judía durante el gobierno de Arturo Alessandri 1932-1938. Revista de estudios histórico-jurídicos, (35), 523-545. https://dx.doi.org/10.4067/S0716-54552013000100016

${ }^{26}$ Sesiones del Senado 26.6.1940, p. 609.

${ }^{27} \mathrm{Ib}$.

${ }^{28}$ Cuadernos Judaicos Núm. 29 (2012): Diciembre 
emigración durante la época del Holocausto debido a que muchos países cerraron sus puertas debido a la depresión económica.

Baldomero Estrada, Académico de la Pontifica Universidad Católica de Valparaíso, considerado un especialista en el tema de migraciones, trabaja en la actualidad, en conjunto con Mario Matus, en la recuperación de la memoria de la Comunidad Israelita de Viña del Mar $^{29}$. Destaca también el articulo Natalio Berman y la inmigración judía a Chile durante la segunda Guerra Mundial, escrito por su nieta Luisa Schonhaut y publicado en Cuadernos Judaicos $\mathrm{N}^{\circ} 32$ (Diciembre, 2015), en él se rescata el rol jugado por este diputado judío comunista, en la política inmigratoria y en la autorización de la llegada de barcos con refugiados judíos al país.

Hay otras áreas en las que es factible encontrar tesis de pregrado de estudiantes de diferentes Facultades. Así es como podemos citar: Judaísmo y espacio cotidiano: El caso de losjudios ashkenazím en Santiago de Chile, de Sharon Kornbluth Nachtygal (Universidad de Chile, 2012). La autora señala que las ciudades están compuestas por un "conjunto de identidades diversas agrupadas en un mismo territorio" y que, según Park $\left(1999\right.$, p.80) ${ }^{30}$, constituyen:

Pequeños mundos que se tocan sin llegar a penetrarse aludiendo a la existencia de grupos segregados que conviven en el espacio urbano, todos tratando de preservar sus formas culturales y mantener sus concepciones individuales y peculiares de la vida, utilizando el espacio compartido de manera diferencial.

Es en este contexto, que Sharon inserta su estudio de judaísmo y espacio cotidiano, refiriéndose especialmente a los judíos ashkenazíes (ashkenazim) que viven en Santiago.

${ }^{29}$ Una primera historia de la formación de dicha comunidad fue escrita hace muchos años por el investigador Moshe Nes El, pero permaneció sin publicar. El texto está en esa Comunidad y una copia del mismo obraba en poder de quien escribe estas notas pero que donó al Archivo Histórico del Judaísmo chileno.

${ }^{30}$ Citado desde la tesis de grado. 
Yeshivá Daat Torá: ciclos de aprendizaje para jóvenes Judios de Chile ${ }^{31}$ de David Lipszyc (Universidad de Chile, 2012), es un proyecto que busca, en palabras de su autor "diseñar un espacio propio dedicado a la cultura judía, para esto es necesario indagar en la cosmovisión y filosofía del judaísmo, y entender los cruces de ideas y establecer una estrategia de aproximación al proyecto de arquitectura, que contemple ambas visiones”.

En el ámbito de espacio y memoria, esta vez desde la perspectiva del arte, destaca la tesis de grado para optar al Grado de Licenciado en Artes con mención en Teoría e Historia del Arte de Carla Steiner (2017) y que lleva el nombre de La persistencia de la Memoria: Holocausto y Monumento ${ }^{32}$. En este trabajo, Steiner se propone analizar las especifidades estéticas que enlazan al monumento con la memoria de la Shoah y, como explicita en la introducción, hará una lectura simbólica tendiente a entender "cómo se plasma en lo visual la memoria colectiva del Holocausto (Shoah), cuál es la intencionalidad memorialística y cómo se resolvió estéticamente en el monumento del Cementerio Israelita de Santiago”.Lo importante de este trabajo consiste en que es el primer estudio relacionado con este tema.

A esos trabajos de jóvenes universitarios se suman la investigación que recupera la memoria de la Filial Fundadora de B'nai Brith en Chile, la Filial Pacifico, fundada en 1936. Esta investigación realizada por Ana María Tapia Adler, Deborah Roitman Meschiani y Judith Riquelme Ríos, lleva por título Los fundadores, Filial Pacifico 235, B'nai B'rith, Distrito 27 y cumple con explorar la génesis y posterior desarrollo de esta Filial, fundadora de la B'nai B'rith en Chile, “cuyos iniciadores y muchos de sus actuales miembros son inmigrantes judios oriundos de Europa Central",33, La historia pudo reconstruirse a partir de entrevistas orales realizadas a sus integrantes gracias a las cuales se pudo conocer datos de su vida en Europa, las dificultades que debieron atravesar, su periplo hasta llegar a Chile y su inserción laboral e ingreso a la Filial. La publicación realizada el año 2016, no contiene las entrevistas, las que se encuentran en el Archivo Histórico del Judaísmo chileno.

${ }^{31}$ repositorio.uchile.cl/handle/2250/112844

${ }^{32}$ Tesis para optar al grado de licenciado en Arte, Facultad de Artes, Universidad de Chile, 2017.

${ }^{33}$ Los Fundadores, p.10. 
El tema de recuperación de la memoria es un proyecto que busca atesorar los escritos publicados o no, de instituciones y comunidades judías como asimismo de judíos que no están afiliados a ninguna de ellos. Los libros Frutos de una sobrevivencia. Fried Vegvari, una Familia con Historia, proveniente de Nyrmada en Hungría y asentada en Viña del Mar donde formaron familia. Rudy Haymann en su libro El tren partió a las 8.30, narra la experiencia que tuvo desde el momento en que como un joven adolescente debió abandonar a su familia y dirigir sus pasos hacia Palestina para salvarse de las garras del nazismo. Lutz Simonsohn, publicó sus memorias el año 2016 en una edición restringida. Se conocen también las memorias aún no publicadas de Saul Locker y Juan Yuhaniak ${ }^{34}$. Todas las mencionadas en este párrafo fueron realizadas fuera del ámbito de la academia y motivadas por la necesidad de recuperar raíces y el deseo de dejar testimonio acerca de sus vivencias tanto de sus lugares de origen como de su inserción en Chile. No cabe duda de que deben existir otras autobiografías, que aún no hemos tenido la posibilidad de conocer. No descartamos la posibilidad de completar este ítem que es importante para la recuperación de la memoria de los judíos en Chile.

Dentro de la misma área de recuperación testimonial, pero desde la Academia, surge la tesis para optar al grado de Magíster en Bibliotecología e Información, de Judith Riquelme, Directora de la Fundación para la Preservación de la Memoria del Judaísmo Chileno y artífice del Archivo Histórico de dicha Fundación; su tesis lleva por título Memoria de refugiados y sobrevivientes de la Shoá en Chile: Resignificación de su pasado a partir de sus archivos e historias de vida. En palabras de la autora de esta tesis, la investigación "se enfocó en conocer la resignificación que los sobrevivientes y refugiados judíos de la Shoá en Chile atribuyen a la migración producida entre los años 1933 a 1950 como consecuencia de la Segunda Guerra Mundial” (p.6). Para ello abordó este trabajo desde la perspectiva fenomenológica y la estrategia de análisis de contenido pues esta metodología

\footnotetext{
${ }^{34}$ Todas ellas se encuentran a disposición del lector en el Archivo Histórico del Judaísmo chileno.
} 
resulta adecuada para esta investigación, ya que se busca analizar el fenómeno desde el punto de vista de las personas y la humanidad. La metodología cualitativa utilizada para realizar la investigación comprendió dos estrategias para obtener información; las historias de vida (entrevistas en profundidad) y la investigación documental que ha permitido complementar la primera con documentos de diferentes archivos oficiales, archivos particulares, y personales.

En lo que respecta a la recuperación de la memoria comunitaria destaca el trabajo serio del Dr. Jacob Cohen quien con gran esfuerzo ha logrado rescatar la historia de la Comunidad Judía de Temuco y de los judíos del sur de Chile. El suyo ha sido un trabajo constante a través del tiempo. De su autoría hay dos obras: Los Judíos de Temuco y Judíos de la Araucanía, esta última un texto que consta de 1200 imágenes entre fotografías y documentos. En la presentación de este libro, se destacó que

No todos los días se tiene la posibilidad de tener en las manos una obra de esta envergadura, verdadero rescate de la historia de una comunidad cuya importancia en la historia del judaísmo chileno es innegable (...) Imprimiendo para la posteridad su historia a través de fotografías y documentos del antes y el ahora, y permitiendo ver el transcurso de los años a través de algunos de sus miembros. ${ }^{35}$

Hay otras dos investigaciones en curso sobre judíos en nuestro país. Ellas son Lo judíos polacos en Chile, a cargo de la socióloga Miriam Krawcyk y la Comunidad Israelita de La Serena de Tapia Adler de la que hubo una presentación preliminar tanto en CIDICSEF (Argentina) como en el Congreso Mundial de Estudios Judaicos realizado en Jerusalem este año. Esta última investigación ha requerido utilizar técnicas de historia oral.

${ }^{35}$ Discurso de presentación del libro realizada por la Prof. Tapia Adler. octubre 16, 2016. 
De anterior data son las publicaciones, Tradición y Adaptación, Vivencia de los Sefaradíes en Chile, de Mario Matus (1993) ${ }^{36}$ correspondiente a su Tesis de Licenciatura y Álbum gráfico y biográfico de los Israelitas en Chile, Valparaíso, de Luis Aguirre (1994) ${ }^{37}$.

En la línea de "institucionalización" de los estudios judaicos, está el Centro de Estudios Judaicos de la Universidad de Chile (CEJ) creado con Decreto $N^{\circ} 6733$ del MINEDUC el 17 de agosto de 1968. Desde sus inicios el centro se ha dedicado al cultivo y desarrollo del conocimiento de la cultura judía y sus proyecciones. A contar de 1970 y hasta 1985, fecha en que el gobierno militar decidió la no dictación de programas conducentes solo a grados, el Centro impartió la Licenciatura en Filosofía con mención en Lengua y Cultura Hebrea, con una duración de 5 años y un Bachillerato en Cultura Hebrea de tres años de duración. Para paliar la pérdida de docencia propia, a contar de 1986 el CEJ realiza docencia en programas de pregrado que imparte la Facultad a la que pertenece y en programas propios de formación en Cultura Judía.

Importante es señalar aquí que el CEJ ha sido, desde su creación, una institución que se ha destacado por ser pionero en el desarrollo de temáticas que integró a las mallas curriculares universitarias tanto en la Facultad a la que está adscrita (Facultad de Filosofía y Humanidades) como en el Departamento de Pregrado de la Vicerrectoría de la Universidad de Chile. Destacamos los Estudios de la Religión, las Culturas del Medio Oriente Antiguo, la creación de Seminarios culturales interdisciplinarios -en épocas en las que el mismo término todavía no era utilizado en nuestra Facultad-, reuniendo en nuestra sede a colegas de distintas universidades y disciplinas y el estudio del Holocausto y los DD.HH, mucho antes de que nuestra universidad creara el Centro de DD.HH. en la Facultad de Derecho (2002) y la cátedra de DD.HH. en Casa Central (2015).

${ }^{36}$ Matus, M (1993). Tradición y Adaptación, Vivencia de los Sefaradies en Chile, Santiago, Departamento de Ciencias Históricas de la Universidad de Chile y Comunidad Israelita Sefaradí de Chile.

${ }^{37}$ Aguirre, L. (1944). Álbum gráfico y biográfico de los Israelitas en Chile, Valparaíso. de Ed. El Mercurio de Valparaíso. 
Hay que destacar, además, que desde sus inicios tuvo una sección dedicada al judaísmo latinoamericano y chileno, tópico en el que llegó a publicar seis volúmenes dedicados íntegramente a dicho tema todos ellos de la autoría del Prof. Günter Böhm (zl). Desde el fallecimiento de este académico, hace ya varios años, esta área no había sido muy trabajada hasta ahora.

La fortaleza del Centro y de lo judaico en dialogo con la sociedad se da en el marco de las actividades docentes y de extensión. El centro imparte cursos de formación general (CFG) destinados a alumnos de todas las carreras de la Universidad de $\mathrm{Chile}^{38}$, cursos básicos transversales (CBT) destinados a alumnos de todas las carreras de la Facultad de Filosofía. $^{39}$ Del mismo modo imparte tres Diplomas de Extensión: Cultura judía, Religiones comparadas y este año se implementó el Diplomado de Shoá, Genocidio y DD.HH. En todos ellos hay una parte en la que se tocan tópicos sobre Judaísmo Chileno y Latinoamericano, si bien no en la profundidad deseada pero si en la medida de lo justo y necesario para tener una noción de la temática.

Puede señalarse que hubo investigación y publicaciones en el área y que, desde esa perspectiva, podría hablarse de una institucionalización de los estudios de judaísmo chileno, al menos en la Universidad de Chile y en ese sentido confiamos en que el creciente interés por las temáticas de inclusión/exclusión, las relaciones entre culturas distintas (pluricultura), las religiones distintas (multirreligiosidad) que coexisten también en nuestro

${ }^{38}$ Los cursos de formación general (CFG) son sobre Multiculturalidad judía, Holocausto y DD.HH, Identidad, Memoria e Historia y Filosofía en tiempos de emergencia, este último a cargo de la Magister en Filosofía, Paula Calderón, que se integró hace un par de años al área de Judaísmo, especialmente a la temática de Pensamiento Judío, área que no había sido trabajada hasta el momento en que ella se integró. Al mismo tiempo se incorporó Nelson Marin, al área de Religiones. ${ }^{39}$ Los cursos básicos transversales (CBT) son Judaísmo, ¿religión o cultura?, Del paraíso al exilio, Los judíos de Sefarad, Holocausto y DD.HH. y un Seminario de Grado sobre Religiones, que se dicta en el Departamento de Historia, ¿Continuidad o ruptura? Del pensamiento bíblico al pensamiento judío moderno. En la Facultad de Artes de la misma Universidad quien escribe dictó entre 1974 y el primer semestre de 2017, muchos cursos relacionados con judaísmo. Entre ellos, Judaísmo y Arte, Shoá a través de la Literatura y el arte, Judaísmo: costumbres y tradiciones a través del cine, Judaísmo: Arte y Cultura, Pueblos y culturas del Medio Oriente Antiguo, El Tanaj a través del arte, solo por mencionar algunos. 
país, sean una vía por la cual transiten algunos de nuestros jóvenes estudiantes y académicos, para ello se cuenta con material que se ha logrado recopilar y que se encuentra en el Archivo Histórico del Judaísmo Chileno.

La creación del Archivo Histórico del Judaísmo Chileno, reconocido a nivel nacional e internacional, forma parte de la Fundación para la preservación de la memoria del judaísmo chileno, entidad creada por Filial Pacífico de B'nai B'rith de Chile, en su deseo de dejar un legado que perdure en el tiempo y sea beneficioso tanto para la comunidad judía como también para el país.

El archivo consta con documentación valiosísima legada por los investigadores Gunter Böhm (zl), Moshé Nes EL (zl) y Jacob Cohen. El acervo documental constantemente está aumentando gracias a donaciones tanto de instituciones como de personas naturales. Dicho material es sometidos a peritaje y salvataje por parte de personas especializadas para después ser digitalizado y subidos a la web del Archivo, todo ello supervisado por su directora Judith Riquelme, bibliotecaria documentalista y especialista en archivística. El Archivo forma parte de la Red Latinoamericana de Archivos Judíos y ha sido reconocido por ICOM, ARPA, y el Archivo Nacional, entidad con la que está en la actualidad trabajando conjuntamente el material desclasificado del Departamento 50 de la Policía de Investigaciones (PDI) que ha permitido conocer detalles del espionaje nazi realizado en nuestro país entre los años 1937 y 1945 y conocido mundialmente como una importante red de contraespionaje durante la II GM que logró desbaratar dos redes de espionaje alemán en nuestro país. Este trabajo conjunto podrá ser visitado en un sitio web que se inaugurará a inicios de enero del año próximo.

¿Podemos afirmar que en Chile pueden considerarse "institucionalizados” los estudios de judaísmo latinoamericano chileno? No lo sé, solo puede afirmarse que es un área en la que se está trabajando y que se está recuperando material valioso. Sin duda queda aún mucho por hacer. 


\section{BIBLIOGRAFIA}

Brahm García, Enrique y Montes Arraztoa, Jorge. El frente popular y la inmigración judía a chile: de la apertura al cierre total. Rev. chil. derecho [online]. 2012, vol.39, n.3 [citado 2017-12-14], pp.891-907. Disponible en:

http://www.scielo.cl/scielo.php?script=sci_arttext\&pid=S0718372012000300020\&lng=es\& nrm=iso>. ISSN 0718-3437. http://dx.doi.org/10.4067/S0718-34372012000300020.

Brahm García, Enrique y Montes Arraztoa, Jorge. (2013). Obstáculos jurídicos y consulares a la inmigración judía durante el gobierno de Arturo Alessandri 19321938. Revista de estudios histórico-jurídicos, (35), 523545. https://dx.doi.org/10.4067/S0716-54552013000100016

Gilberto Aranda y Sergio Salinas (editores), Identidades judías y sionistas en conflicto. Diásporas judías en el Cono Sur americano.

Lorenzo Agar y Abraham Magendzo: Diálogo intercultural. Caso Comunidad Judía y árabe en Chile. Santiago: Fundación Ideas, Proyecto DIJO, pp. 185-212. Proyecto FONDECYT N 1050053.

Navarro-Rosenblatt, V. Ampliando los límites de la integración social: género e historia judía contemporánea, Cuadernos Judaicos, Centro de Estudios Judaicos, Universidad de Chile, 30 (2013).

Revista Cuadernos Judaicos ISSN: 0718-8749 N²5 (2008), `o26 (2009), ํo27 (2010), $\mathrm{N}^{\circ} 30$ (2013), $\mathrm{N}^{\circ} 31$ (2014), $\mathrm{N}^{\circ} 32(2015)$.

Sznajder, M. Mapocho, 1992. 
Sznajder, M. Estudios Interdisciplinarios de América Latina y el Caribe $(1,1,1990) \mathrm{pp}$. 41-57.

Sznajder, M. Yahadut Zmaneinu (7, 1992) pp. 311-327. (Hebrew).

Sznajder, M., en M. L. Tucci Carneiro (ed.) O Anti-semitismo nas Américas (Sao Paulo: Editora EDUSP, 2008) pp. 437-457.

Sznajder, M., Judaica Latinoamericana $(2,1993)$ pp. 137-148. 